\title{
Acoustical cross-talk in row-column addressed 2-D transducer arrays for ultrasound imaging
}

Christiansen, Thomas Lehrmann; Jensen, Jørgen Arendt; Thomsen, Erik Vilain

Published in:

Ultrasonics

Link to article, DOI:

10.1016/j.ultras.2015.07.008

Publication date:

2015

Document Version

Peer reviewed version

Link back to DTU Orbit

Citation (APA):

Christiansen, T. L., Jensen, J. A., \& Thomsen, E. V. (2015). Acoustical cross-talk in row-column addressed 2-D transducer arrays for ultrasound imaging. Ultrasonics, 63, 174-178. https://doi.org/10.1016/j.ultras.2015.07.008

\section{General rights}

Copyright and moral rights for the publications made accessible in the public portal are retained by the authors and/or other copyright owners and it is a condition of accessing publications that users recognise and abide by the legal requirements associated with these rights.

- Users may download and print one copy of any publication from the public portal for the purpose of private study or research.

- You may not further distribute the material or use it for any profit-making activity or commercial gain

- You may freely distribute the URL identifying the publication in the public portal 


\title{
Acoustical cross-talk in row-column addressed 2-D transducer arrays for ultrasound imaging
}

\author{
Thomas Lehrmann Christiansen ${ }^{\mathrm{a}, *}$, Jørgen Arendt Jensen ${ }^{\mathrm{b}}$, Erik Vilain Thomsen ${ }^{\mathrm{a}}$ \\ ${ }^{a}$ Department of Micro- and Nanotechnology, Technical University of Denmark, Building 345E, DK-2800 Kgs. Lyngby, Denmark \\ ${ }^{b}$ Center for Fast Ultrasound Imaging, Department of Electrical Engineering, Technical University of Denmark, Building 349, DK-2800 Kgs. Lyngby, Denmark
}

\begin{abstract}
The acoustical cross-talk in row-column addressed 2-D transducer arrays for volumetric ultrasound imaging is investigated. Experimental results from a $2.7 \mathrm{MHz}, \lambda / 2$-pitch capacitive micromachined ultrasonic transducer (CMUT) array with 62 rows and 62 columns are presented and analyzed in the frequency-wavenumber domain. The sources of cross-talk are identified and predicted theoretically. The nearest neighbor cross-talk is $-23.9 \pm 3.7 \mathrm{~dB}$ when the array is used as a 1-D array with the rows functioning as both transmitters and receivers. In the row-column configuration, with the columns transmitting and the rows receiving, the cross-talk is reduced to $-40.2 \pm 3.5 \mathrm{~dB}$.
\end{abstract}

Keywords: Cross-talk, 2-D transducer array, Row-column, CMUT, Volumetric ultrasound imaging

\section{Introduction}

Row-column addressed 2-D transducer arrays have been shown to provide volumetric ultrasound images utilizing a significantly reduced number of connections to the array compared to 2-D matrix arrays [1, 2, 3, 4]. The elements are addressed by their row- and column indices, effectively creating two orthogonal 1-D arrays, where typically the columns are used as transmitters and the rows as receivers or vice versa. However, the acoustical cross-talk in such arrays have so far not been investigated. This paper addresses this subject using experimental data from a $2.7 \mathrm{MHz}, \lambda / 2$-pitch capacitive micromachined ultrasonic transducer (CMUT) array with 62 rows and 62 columns. This specific array was originally developed for volumetric medical imaging and incorporates an integrated apodization to reduce ghost echoes in the beamformed image. The array center frequency was selected to allow volumetric imaging down to a depth of $10-15 \mathrm{~cm}$, and the number of elements were chosen to provide a reasonable total footprint size of the transducer array $(26.3 \mathrm{~mm} \times 26.3 \mathrm{~mm})$. Further details on the layout, fabrication, and imaging performance of the array are given in two previous publications [2, 3]. In this study, the array is used solely for investigating the acoustical cross-talk effects in row-column addressed CMUT arrays.

Several studies involving 1-D linear CMUT arrays for medical imaging have revealed significant acoustical cross-talk in such arrays, reporting nearest neighbor cross-talk on the order of $-20 \mathrm{~dB}$ relative to the emitting element $[5,6]$. The origin of the cross-talk has been identified as being Lamb waves in the silicon substrate, interface waves, and the longitudinal waves emitted into the medium [5, 6, 7]. Of these, the by far most dominating contribution are the interface waves [6]. Using a

${ }^{*}$ Corresponding author, e-mail: tlehr@nanotech.dtu.dk row-column addressed CMUT array presented in [2, 3], it is here shown that the orthogonal arrangement of transmitter- and receiver elements in the row-column addressing scheme averages out the majority of the cross-talk, which is a previously unknown advantage of such arrays. Similar to previous investigations for 1-D CMUT arrays, the cross-talk is measured by emitting with one element and recording the signal on all receiving elements [6]. This yields data in the temporal-spatial domain, which is subsequently analyzed in the frequency domain using a 2-D Fourier transform of the data [8].

The cross-talk cancelling effect of the row-column addressing scheme is illustrated through two experiments: In the first experiment, one row is emitting and all rows are receiving as shown in Fig. 1 (left). This mimics the configuration in a 1-D array used for 2-D B-mode imaging. In the second experiment, one column is emitting while all rows are receiving, see Fig. 1 (right). This corresponds to the row-column configuration used for volumetric imaging. It is shown that the 1-D configuration yields results consistent with previous cross-talk investigations with 1-D CMUT arrays [6], while the row-column configuration exhibits significantly reduced cross-talk levels.

This paper is organized as follows: First, the dispersion relations of the expected types of cross-talk waves are presented using idealizing assumptions. Then, the experimental setup used to measure the cross-talk is described. The results are subsequently presented both in the temporal-spatial domain and in the frequency domain. Finally, the latter is used to identify the cross-talk mechanisms in the array by comparing it to the derived dispersion relations.

\section{Dispersion relations}

As briefly covered above, the acoustical cross-talk in a CMUT array is in general comprised of substrate waves, in- 


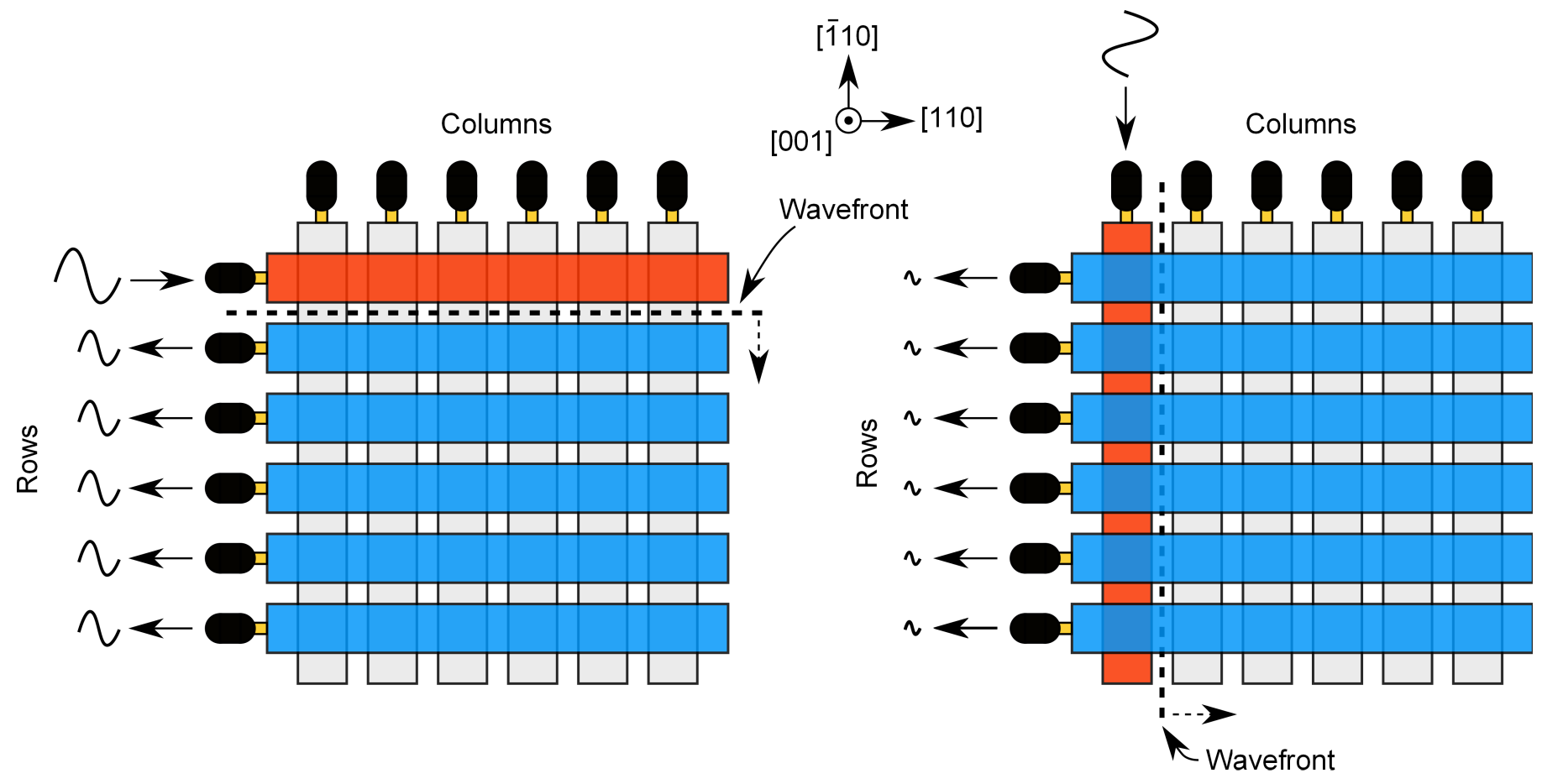

Figure 1: Illustration of the two setups used for measuring the cross-talk. In the case to the left, one row (red) is actuated, and the received signal on all rows (blue) are recorded. This corresponds to a 1-D array configuration. In the case to the right, one column is actuated, and the signals from the rows are recorded. This corresponds to the row-column configuration. The wavefront generated by the actuated element is shown with a black dashed line. The size of the sine-symbols graphically illustrates the magnitude of the signals. The crystallographic directions of the silicon substrate is shown in the top.

terface waves, and longitudinal waves in the medium. While the first two are dispersive waves, the latter are travelling nondispersively at the speed of sound $c$ in the medium. The following gives a brief presentation of the dispersion relation for the substrate waves and the interface waves.

The substrate of the CMUT is made from a silicon wafer, and the waves travelling in this may be described by Lamb wave theory. In practice, the silicon substrate will be supported and have finite lateral extent, which may contribute to attenuation and mode conversions of the substrate waves. However, in the interest of simplicity, an idealized case is assumed in which the substrate is an isolated silicon plate of thickness $d$ having infinite lateral extent. In this case, a pair of characteristic equations describe the dispersion relation of the Lamb waves [9]:

$$
\begin{aligned}
& \frac{\tan (\beta d / 2)}{\tan (\alpha d / 2)}=-\frac{4 \alpha \beta k^{2}}{\left(k^{2}-\beta^{2}\right)^{2}} \\
& \frac{\tan (\beta d / 2)}{\tan (\alpha d / 2)}=-\frac{\left(k^{2}-\beta^{2}\right)^{2}}{4 \alpha \beta k^{2}}
\end{aligned}
$$

Here, $\alpha^{2}=\omega^{2} / c_{L}^{2}-k^{2}$ and $\beta^{2}=\omega^{2} / c_{T}^{2}-k^{2}$ where $\omega$ is the angular frequency, and $k$ is the wave number. $c_{L}$ and $c_{T}$ are the longitudinal and shear wave velocities, respectively. In this work, the substrate is a (001) silicon wafer having cubic symmetry along its principal axes with stiffness coefficients $c_{11}=165.64 \mathrm{GPa}$, $c_{12}=63.94 \mathrm{GPa}$, and $c_{44}=79.51 \mathrm{GPa}$ [10]. The transducer array is aligned to the wafer flat, which indicates the [110] crystal orientation. Thus, a Lamb wave propagating away from the emitting element travels in the $\langle 110\rangle$-directions as shown in Fig. 1. It follows that $c_{L}=\sqrt{\left(c_{11}+c_{12}+2 c_{44}\right) /(2 \rho)}=$ $9132 \mathrm{~m} / \mathrm{s}$ and $c_{T}=\sqrt{c_{44} / \rho}=5842 \mathrm{~m} / \mathrm{s}$ [11], where $\rho=$ $2330 \mathrm{~kg} / \mathrm{m}^{3}$ is the mass density of silicon.

Equation (1) describes the symmetric modes, $S_{n}$, whereas Eq. (2) describes the antisymmetric modes, $A_{n}$. The zerothorder modes exist for all frequencies and have been observed in several studies of 1-D CMUT arrays [5, 6]. The higher-order modes have lower cut-off frequencies at $f_{c}=n c_{L} /(2 d)$ and $f_{c}=n c_{T} /(2 d)$. At these frequencies, the plate resonates with infinite phase velocity and zero group velocity. Ladabaum et al. observed significant substrate ringing at the frequencies corresponding to longitudinal-wave resonances along the thickness of the substrate, and demonstrated that these resonances could be moved out of the transducer frequency spectrum by thinning the substrate [12].

In addition to substrate waves and waves in the medium, evanescent waves may propagate next to the interface between the transducer and the medium. Previous studies have shown that such waves are the most significant contribution to acoustical cross-talk in 1-D CMUT arrays [5, 6]. Eccardt et al. have provided a simple description of the dispersion relation of these waves in CMUT arrays based on the assumption of a semiinfinite homogeneous fluid interfaced by a semi-infinite solid with a given surface stiffness per area and mass per area [7]. In this framework, the pressure wave propagating in the fluid at the interface is described by $p=\exp (-\gamma z+j k x-j \omega t)$ with $\gamma>0$ being the decay constant in the $z$-direction perpendicular to the surface. Denoting the speed of sound in the medium $c$, the wave number along the surface is given by the relation 
$k^{2}=\gamma^{2}+\omega^{2} / c^{2}$, which is found by inserting the expression for the pressure in the time-dependent wave equation for the medium, $\partial^{2} p / \partial t^{2}=c^{2} \nabla^{2} p$. The impedance of the surface wave is given by $Z_{w}=j \omega \rho_{m} / \gamma$, where $j$ is the imaginary unit and $\rho_{m}$ is the mass density of the medium [7]. A surface with a homogeneous stiffness per area $s$ and mass per area $m$ will have an acoustic impedance given by $Z_{i}=s /(j \omega)+j \omega m$. The wave travels along this surface when $Z_{w}=Z_{i}$, and thus $\gamma=j \omega \rho_{m} / Z_{i}$. It therefore follows that the phase velocity of the surface wave is

$$
c_{\text {phase }}=\frac{\omega}{k}=\frac{c}{\sqrt{1-\left(\frac{\rho_{m} c}{Z_{i}}\right)^{2}}},
$$

from which the dispersion relation $k=\omega / c_{\text {phase }}$ is readily found. Note that for an infinitely stiff surface, the interface wave propagates non-dispersively at $c_{\text {phase }}=c$.

\section{Experimental method}

The transducer used in this work is a CMUT array with 62 rows and 62 columns. The elements have a mean center frequency of $2.7 \mathrm{MHz}$ and a pitch of $270 \mu \mathrm{m}$. Each element consists of densely packed square CMUT cells with a side length of $60 \mu \mathrm{m}$ and an inter-distance of $5 \mu \mathrm{m}$. Further details can be found in a previous publication [3].

The transducer array is mounted and wire-bonded on a printed circuit board with each of the 124 channels connected to the SARUS research scanner system [13]. In order to drive the cable to the scanner system, the received signals are amplified though MAX4805A pre-amplifiers (Maxim Integrated, San Jose, CA, USA) providing a nominal $8.7 \mathrm{~dB}$ voltage gain. All elements are kept at a constant DC voltage of $80 \mathrm{~V}$. The transducer array is then mounted in a sealed box that allows it to be submerged in vegetable oil containing no reflecting structures. Vegetable oil is used to avoid short circuiting of the array, which does not have a protective coating nor lens.

In the first experiment, only the rows of the array are used in order to mimic a 1-D array. The setup is illustrated in Fig. 1 (left). The outer-most row element is actuated using a 4-cycle, $2.5 \mathrm{MHz}$ AC voltage signal with an amplitude of $50 \mathrm{~V}$ on top of the $80 \mathrm{~V} \mathrm{DC}$ bias. The onset of the AC signal triggers the scanner system, which then records the voltage signal on all the rows for a duration of $54 \mu \mathrm{s}$. At a speed of sound in the vegetable oil of $1480 \mathrm{~m} / \mathrm{s}$, this corresponds to an imaging depth of $4 \mathrm{~cm}$.

The second experiment is illustrated in Fig. 1 (right). Here, the setup is the same as in the first experiment, except that this time the outer-most column element is actuated. This therefore corresponds to the situation where the array is used in the row-column configuration with transmitting elements oriented orthogonal to receiving elements.

\section{Results and discussion}

The result of the two experiments are shown in Fig. 2, the $x$-axis being the position of the receive element and the $y$-axis being time. Both plots are normalized to the maximum value in the 1-D configuration. The black dashed line indicates the end of the transmit pulse and ring-down of the electronics at $2.8 \mu \mathrm{s}$, which saturates the receivers on all channels. This saturation is seen as a uniform signal for all positions. Data recorded earlier than $2.8 \mu$ s are therefore not included in the subsequent analysis.

In the data from the 1-D configuration (Fig. 2, left), the emitting element is located to the far left in the figure. High-velocity waves are visible in the upper right corner, but the most dominating waves occur at varying velocities and are seen to experience little damping. Also, reflections from the array edge can be seen. The nearest neighbor cross-talk in the 1-D configuration was found by repeating the measurement sequence with a new emitting row element for each sequence, yielding a mean of $-23.9 \mathrm{~dB}$ with a standard deviation of $-3.7 \mathrm{~dB}$ consistent with results in the literature $[5,6]$. The nearest neighbor cross-talk was calculated by recording the signal from the neighbor to the emitting element and normalizing it to the transmit voltage of the emitting element corrected for the insertion loss of the emitting element.

From the data acquired in the row-column configuration (Fig. 2, right), it is evident that the amplitude of the cross-talk is significantly reduced compared to the 1-D configuration. In the row-column configuration, the concept of nearest neighbor cross-talk does not make sense due to the orthogonal orientation of transmit- and receive elements. Instead, the average of the maximum signal received on each receiving element is used. Repeating the emission sequence for each of the columns and recording on all rows for each emission yields a mean cross-talk of $-40.2 \mathrm{~dB}$ with a standard deviation of $3.5 \mathrm{~dB}$.

To further analyze the data, a 2-D Fourier transform is performed on the data for times greater than $2.8 \mu$ s [8]. The data is multiplied with a Hann-window in both dimensions to suppress side-lobes. The resulting plots in the frequency domain are shown in Fig. 3. The maximum spatial frequency corresponds to one-half of the reciprocal pitch of the transducer array (the Nyquist frequency). The maximum temporal frequency is set to $4 \mathrm{MHz}$, since no data is within the dynamic range of the plot above this frequency. The dispersion curves for the interface waves, substrate waves, and longitudinal waves in the medium have been plotted as well using the theory presented in Section 2. Calculation of the Lamb wave dispersion curves was done using the array substrate thickness of $520 \mu \mathrm{m}$. Only the zeroth-order modes are visible in the shown frequency range. The dispersion curve for the interface waves was calculated assuming that the whole transducer surface is covered by CMUT cells (i.e. neglecting the $5 \mu \mathrm{m}$ wide anchoring area between the cells). The surface stiffness per area is then found as the stiffness of a single cell divided by its effective area. For a square cell with a plate thickness of $h=2 \mu \mathrm{m}$ and a side-length of $2 L=60 \mu \mathrm{m}$, the stiffness is $s_{\text {cell }}=771.3 \mathrm{GPa} h^{3} / L^{2}$ and the effective area is $a_{\text {eff }}=1.215 L^{2}$ [14]. The mass per area of the surface is simply $m=\rho h$.

Note that both substrate waves, interface waves, and waves in the medium are clearly observed for the 1-D configuration (Fig. 3, left). The plot has been normalized to the amplitude of 

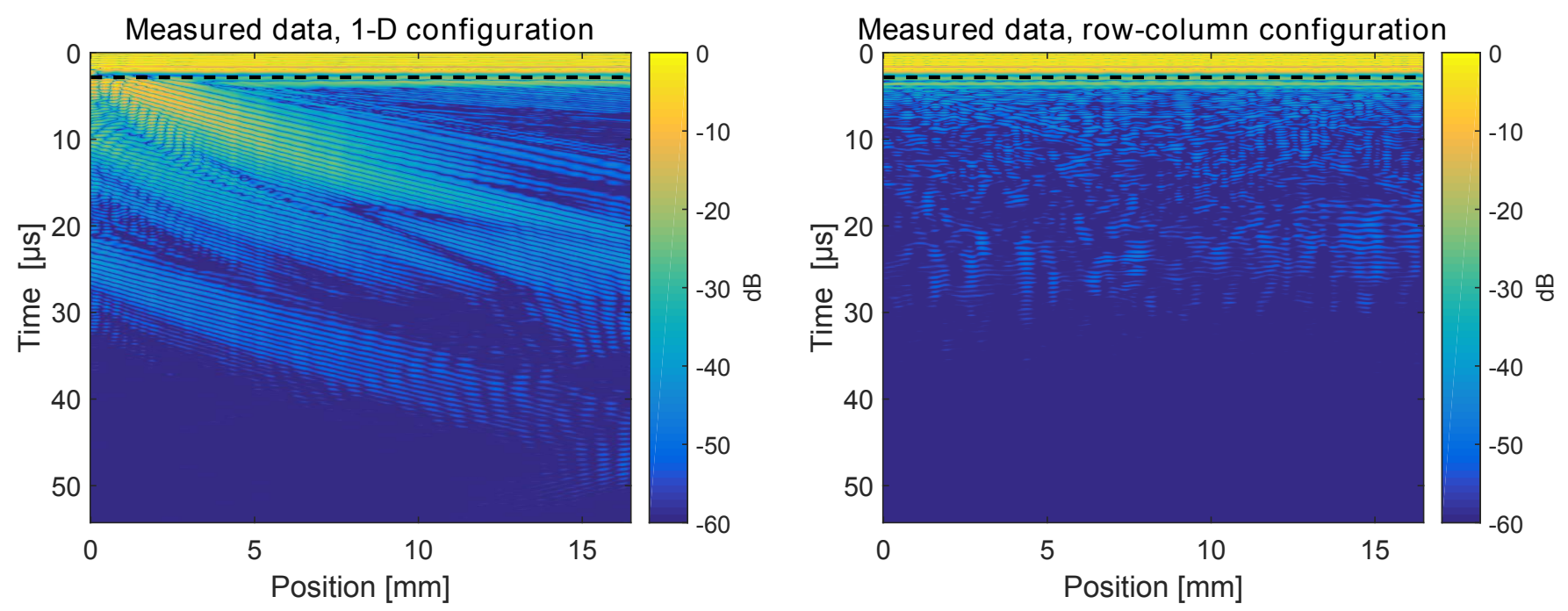

Figure 2: Data acquired using the two setups shown in Fig. 1. Time zero corresponds to the onset of the transmit pulse. The black dashed line indicates the end of the transmit pulse, and only data after this time has been included in the cross-talk analysis.
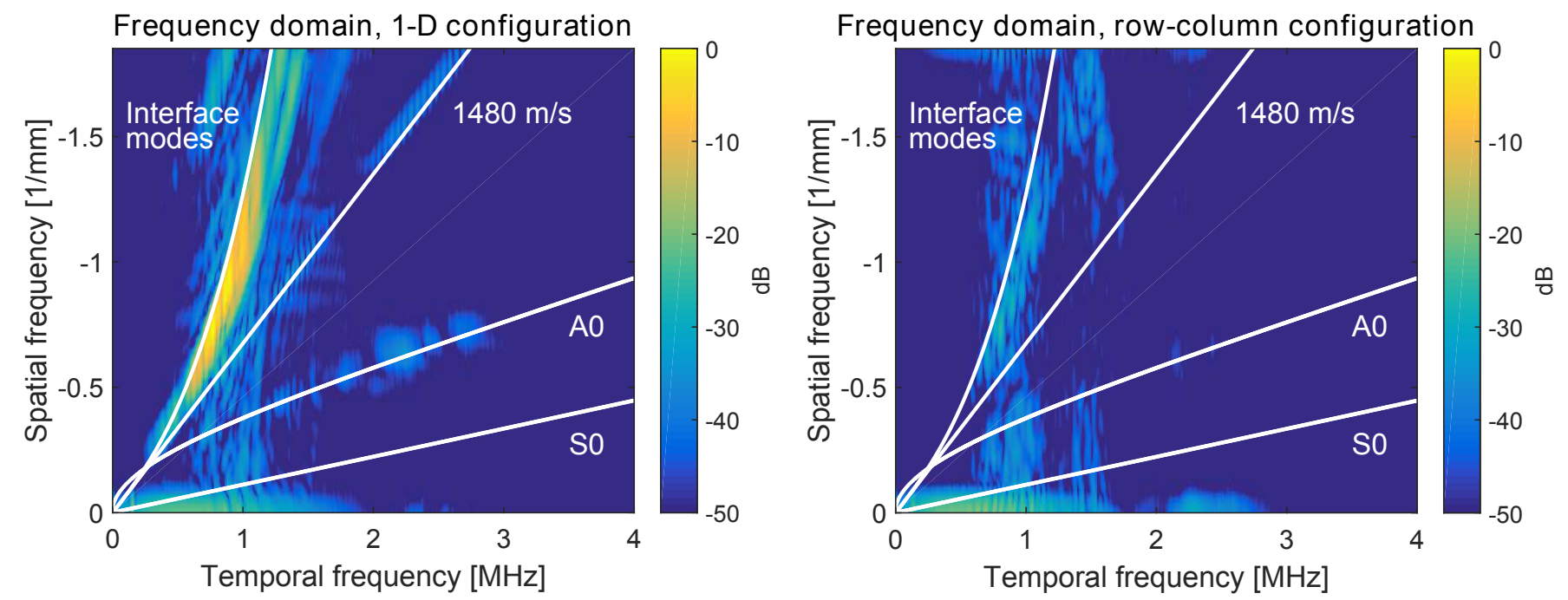

Figure 3: 2-D Fourier transforms of the data shown in Fig. 2. The data has been multiplied with a Hann-window in both the spatial and temporal dimension before the Fourier transform. The theoretically calculated dispersion curves for the interface modes, the waves in the medium, and the substrate Lamb waves are shown as white solid lines. Both plots are normalized to the maximum value in the 1-D configuration. 
the interface waves, which are by far the most dominating. The non-dispersive waves in the medium are located at or more than $36 \mathrm{~dB}$ below the interface waves, while the number is $34 \mathrm{~dB}$ for the $A_{0}$ Lamb wave. The $S_{0}$ Lamb wave is not visible within the displayed dynamic range. The substrate waves and the waves in the medium are most visible in the frequency range defined by the $-6 \mathrm{~dB}$ bandwidth of the transducer, which has its limits at $0.95 \mathrm{MHz}$ and 3.4 MHz, respectively [3]. The interface modes have their maximum frequency response at $0.9 \mathrm{MHz}$, which is outside the transducer bandwidth. This lower frequency for the interface modes was also observed by Bayram et al. [6]. They identified the frequency shift as being due to the difference in center frequency of the CMUT cells of the transmitting element, which are actuated in-phase, and the CMUT cells of the receiving elements, which are actuated out of phase as a consequence of the propagating interface wave.

Despite the idealizing assumptions used to derive the theoretical dispersion curves, all types of waves are identified by the theory. The dispersion curve for the interface modes would follow the non-dispersive line at $1480 \mathrm{~m} / \mathrm{s}$ for an infinitely stiff transducer surface [7]. Since the actual surface is comprised of small areas of solid silicon in addition to the CMUT cells, the calculated dispersion curve expectedly borders the lower part of the interface modes' spectrum.

For the row-column configuration (Fig. 3, right), the crosstalk is seen to be reduced by more than $24 \mathrm{~dB}$. The plot is normalized to the $0 \mathrm{~dB}$-value for the $1-\mathrm{D}$ configuration to illustrate the reduction. The reason for this is the orientation of the receiving elements relative to the emitting elements. As illustrated in Fig. 1 (left), the wave front of the acoustical wave generated by the emitting element in the 1-D configuration will impinge parallel to each of the receiving elements. Thus, the whole length of the element surface vibrates in phase, generating a strong signal. The case for the row-column configuration is seen in Fig. 1 (right). Here, the wave front from the emitting element is oriented perpendicular to the receiving elements. The element is considerably longer than the wavelength of the acoustical wave, corresponding to $\simeq 30 \lambda$ at the transducer center frequency of $2.7 \mathrm{MHz}$ and $\simeq 10 \lambda$ at the main frequency response of the dominating interface waves $(0.9 \mathrm{MHz})$. Therefore, the signals generated at the actuated parts of the element will average out due to the symmetry of the wave, and ideally no signal is received. In practice, however, the finite length of the elements and the reflections and mode conversions of the waves will contribute with some net cross-talk as observed in the experiment.

\section{Conclusion}

This paper investigated the acoustical cross-talk in a rowcolumn addressed CMUT array for ultrasound imaging. It was shown that when used as a 1-D array, the array exhibited a cross-talk of $-23.9 \pm 3.7 \mathrm{~dB}$, consistent with previous results in the literature. The acoustical cross-talk was identified as Lamb waves in the substrate, interface waves, and nondispersive waves in the medium. When used in the row-column configuration, with columns emitting and rows receiving, the cross-talk was reduced to $-40.2 \pm 3.5 \mathrm{~dB}$ due to the orthogonal orientation of the transmit- and receive elements.

\section{Acknowledgments}

The authors would like to thank Morten Fischer Rasmussen for assistance with setting up the experiment, and BK Medical ApS (Herlev, Denmark) for producing the interface electronics. This work is financially supported by the Danish National Advanced Technology Foundation (82-2012-4).

\section{References}

[1] C. H. Seo, J. T. Yen, A 256 x 2562 -D array transducer with row-column addressing for 3-D rectilinear imaging, IEEE Trans. Ultrason., Ferroelec., Freq. Contr. 56 (4) (2009) 837-847.

[2] M. F. Rasmussen, T. L. Christiansen, E. V. Thomsen, J. A. Jensen, 3-D imaging using row-column-addressed arrays with integrated apodization — part i: Apodization design and line element beamforming, IEEE Trans. Ultrason., Ferroelec., Freq. Contr. 62 (5) (2015) 947-958.

[3] T. L. Christiansen, M. F. Rasmussen, J. P. Bagge, L. N. Moesner, J. A. Jensen, E. V. Thomsen, 3-D imaging using row-column-addressed arrays with integrated apodization - part ii: Transducer fabrication and experimental results, IEEE Trans. Ultrason., Ferroelec., Freq. Contr. 62 (5) (2015) 959-971.

[4] R. K. W. Chee, A. Sampaleanu, D. Rishi, R. J. Zemp, Top orthogonal to bottom electrode (TOBE) 2-D CMUT arrays for 3-D photoacoustic imaging, IEEE Trans. Ultrason., Ferroelec., Freq. Contr. 61 (8) (2014) 1393-1395.

[5] X. Jin, Ö. Oralkan, F. L. Degertekin, B. T. Khuri-Yakub, Characterization of one-dimensional capacitive micromachined ultrasonic immersion transducer arrays, IEEE Trans. Ultrason., Ferroelec., Freq. Contr. 48 (3) (2001) 750-760.

[6] B. Bayram, M. Kupnik, G. G. Yaralioglu, Ö. Oralkan, A. S. Ergun, D.-S. Lin, S. H. Wong, B. T. Khuri-Yakub, Finite element modeling and experimental characterization of crosstalk in 1-d CMUT arrays, IEEE Trans. Ultrason., Ferroelec., Freq. Contr. 54 (2) (2007) 418-430.

[7] P.-C. Eccardt, A. Lohfink, H.-G. von Garssen, Analysis of crosstalk between fluid coupled CMUT membranes, in: Proc. IEEE Ultrason. Symp., 2005, pp. 593-596.

[8] D. Alleyne, P. Cawley, A two-dimensional Fourier transform method for the measurement of propagating multimode signals, J. Acoust. Soc. Am. 89 (3) (1991) 1159-1168.

[9] H. Lamb, On waves in an elastic plate, Proc. Roy. Soc. (London), Ser. A 93 (1917) 114-128.

[10] J. J. Hall, Electronic effects in the elastic constants of n-type silicon, Phys. Rev. 161 (3) (1967) 756-761.

[11] G. F. Miller, M. J. P. Musgrave, On the propagation of elastic waves in aeolotropic media. III. media of cubic symmetry, Proc. Roy. Soc. (London), Ser. A 236 (1956) 352-383.

[12] I. Ladabaum, P. Wagner, C. Zanelli, J. Mould, G. Wojcik, Silicon substrate ringing in microfabricated ultrasonic transducers, in: Proc. IEEE Ultrason. Symp., 2000, pp. 943-946.

[13] J. A. Jensen, H. Holten-Lund, R. T. Nilsson, M. Hansen, U. D. Larsen, R. P. Domsten, B. G. Tomov, M. B. Stuart, S. I. Nikolov, M. J. Pihl, Y. Du, J. H. Rasmussen, M. F. Rasmussen, SARUS: A synthetic aperture realtime ultrasound system, IEEE Trans. Ultrason., Ferroelec., Freq. Contr. 60 (9) (2013) 1838-1852.

[14] M. F. la Cour, T. L. Christiansen, J. A. Jensen, E. V. Thomsen, Electrostatic and small-signal analysis of CMUTs with circular and square anisotropic plates, IEEE Trans. Ultrason., Ferroelec., Freq. Contr. (2015) in press. 